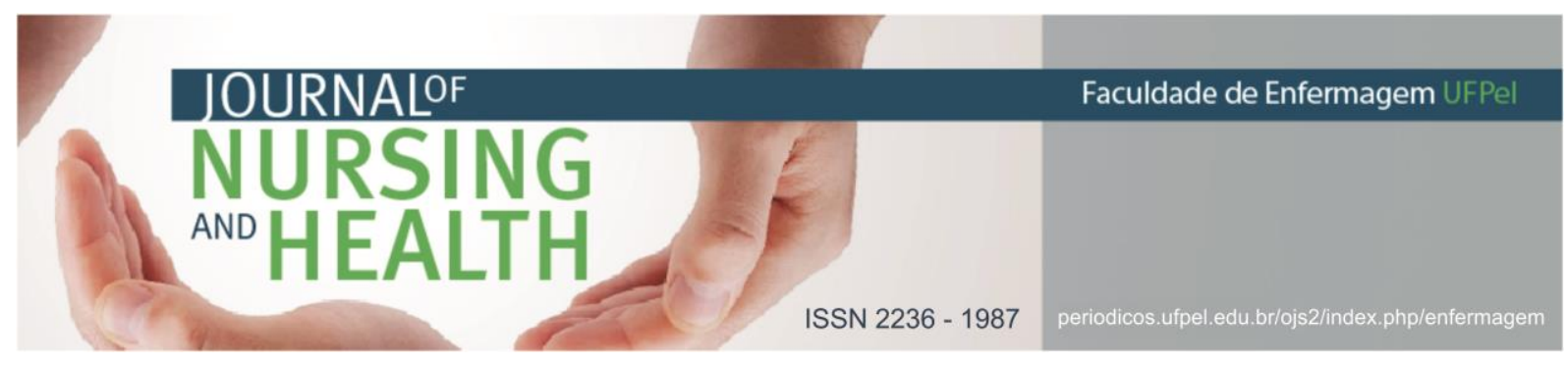

ARTIGO DE REVISÃO

\title{
O uso da música nos serviços de saúde: uma revisão integrativa
}

\author{
The use of music in the health services: an integrative review
}

\author{
El uso de la música en los servicios de salud: una revisión integrativa
}

Santee, Kadija Mohamed ${ }^{1}$; Oliveira, Tarley Santos²; Santos, Thatyane Rosa ${ }^{3}$; Lima, Maíra Ribeiro Gomes $^{4}$; Fernandes, Carla Natalina da Silva 5 ; Pilger, Calíope ${ }^{6}$

Como citar este artigo: Santee KM, Oliveira TS, Santos TR, Lima MRG, Fernandes CNS, Pilger C. 0 uso da música nos serviços de saúde: uma revisão integrativa J. nurs. health. 2019;9(2):e199201

\section{RESUMO}

Objetivo: analisar e sintetizar informações quanto a utilização da música como estratégia de cuidado pelos profissionais que trabalham nos serviços de saúde. Métodos: trata-se de uma Revisão Integrativa de Literatura, nas bases de dados: PubMed, Scientific Electronic Library Online e Literatura LatinoAmericana e do Caribe em Ciências da Saúde, que buscou responder à pergunta: nos serviços de saúde, a música tem sido utilizada como estratégia de cuidado pelos profissionais? Foram analisados 34 trabalhos e emergiram quatro categorias. Resultado: a musicoterapia mostrou ser benéfica para a diminuição da dor, da ansiedade, de sintomas comportamentais em pacientes psiquiátricos e no aumento do aleitamento materno. Conclusões: os principais setores da saúde que utilizaram a música como terapia foram as obstétricas, cirúrgicas e as psiquiátricas, com o intuito de acolher o paciente de uma forma humanizada e prover uma melhor qualidade de vida dentro das circunstâncias.

Descritores: Música; Terapias complementares; Serviços de saúde.

\section{ABSTRACT}

Objective: analyze and synthesize information about the use of music as strategy of care by the professionals in health services. Methods: this article is an Integrative Review of the literature where electronic databases were used, such as: PubMed, Scientific Electronic Library Online and Literatura Latino-Americana e do Caribe em Ciências da Saúde to answer the question: in the health services, has the music been used as one strategy of care by the professionals? Thirty-four articles were analyzed, and four categories emerged. Results: the music therapy showed to be benefic in decreasing pain, anxiety, behavior symptoms in psychiatric patients and increasing breast feeding. Conclusion: the main sectors of health that used music as therapy were the obstetric, surgical and psychiatric ones, with the intention of welcoming the patient in a humanized way and providing a better quality of life under the circumstances.

Descriptors: Music; Complementary therapies; Health services.

1 Enfermeira. Universidade de São Paulo (USP). E-mail: kadija.santee@gmail.com http://orcid.org/0000-00020245-7325

2 Enfermeiro. Universidade Federal de Goiás (UFG). E-mail: tarley13@live.com http: //orcid.org/0000-0002-22180116

3 Enfermeira. Universidade Federal de Goiás (UFG). E-mail: thatyane96@hotmail.com http://orcid.org/00000002-3407-6391

4 Enfermeira. Especialista em Enfermagem Obstétrica. Universidade Federal de Goiás (UFG). E-mail: mairaribeiroenf@gmail.com http: //orcid.org/0000-0003-0570-9281

5 Enfermeira. Doutora em Ciências. Universidade Federal de Goiás (UFG). E-mail: carla_natalina@yahoo.com.br http: / / orcid.org/0000-0002-0832-4830

6 Enfermeira. Doutora em Ciências. Universidade Federal de Goiás (UFG). E-mail: caliopepilger@hotmail.com http: / /orcid.org/0000-0002-1017-6099 


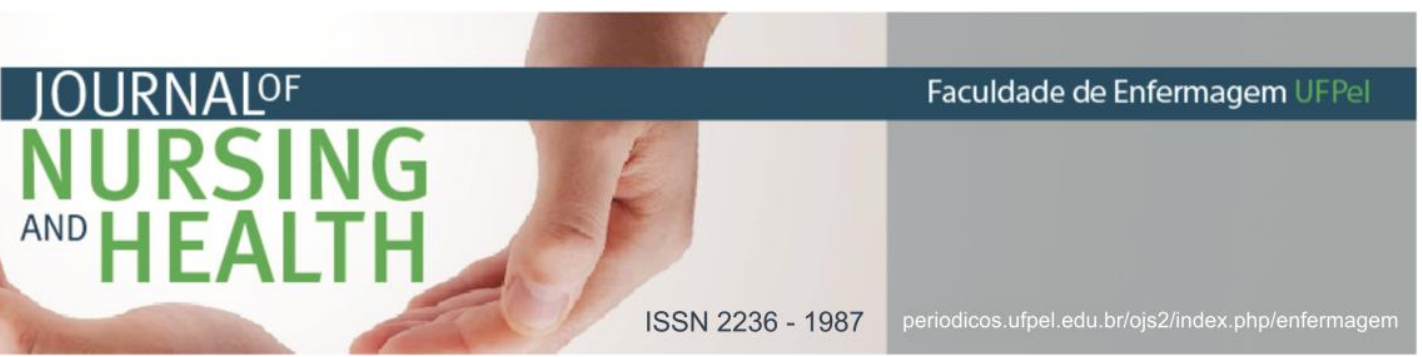

RESUMEN

Objetivo: analizar y sintetizar informaciones sobre la utilización de la música como estrategia de cuidado por los profesionales que trabajan em los servicios de salud. Métodos: se trata de una Revisión Integrativa, em las bases de datos: PubMed, Scientific Electronic Library Online, Literatura Latino-Americana e do Caribe em Ciências da Saúde, que buscó responder: ¿en los servicios de salud la música ha sido utilizada como estrategia de cuidado por los profesionales? Se analizaron 34 trabajos y emergieron cuatro categorías. Resultados: la musicoterapia se mostró beneficiosa para disminuir el dolor, la ansiedad, los síntomas conductuales en los pacientes psiquiátricos y el aumento de la lactancia materna. Conclusiones: los principales sectores de salud que utilizaron la música como terapia fueron las obstétricas, quirúrgicas y las psiquiátricas, con el propósito de acoger al paciente de una forma humanizada y proveer una mejor calidad de vida dentro de las circunstancias. Descriptores: Música; Terapias complementarias; Servicios de salud.

\section{INTRODUÇÃO}

No Brasil a Política Nacional de Práticas Integrativas

e

Complementares (PNPIC) instituída pela portaria do Ministério da Saúde (MS) $\mathrm{n}^{\circ} 971$ em 3 de maio de 2006 incluiu no Sistema Único de Saúde (SUS) práticas de saúde como a medicina tradicional chinesa/acupuntura, plantas medicinais e fitoterapia, homeopatia, medicina antroposófica e termalismo social/crenoterapia. ${ }^{1}$ Essas terapias apresentaram nos últimos anos grande crescimento e utilização pelos serviços de saúde pública, sendo que a Organização Mundial de Saúde (OMS) reconhece e incentiva sua aplicação. ${ }^{1}$

Após dez anos da criação da lei instituída pela portaria do MS $n^{\circ} 971$, o Brasil possui aproximadamente 5.000 locais que realizam e ofertam as Práticas Integrativas e Complementares (PIC). Estas instituições são de diversas complexidades nas quais $78 \%$ se apresentam na Atenção Básica, 18\% em serviços especializados e $4 \%$ na atenção hospitalar. A portaria $n^{\circ}$ $849 / 2017$ reconhece e adiciona outras PIC aos serviços de saúde, dentre elas podemos elencar: Musicoterapia,
Dança Circular, Arteterapia, Reflexoterapia, Naturopatia, Reiki, Shantala, Ayurveda, Yoga, Biodança, Quiropraxia, Meditação, Terapia Comunitária e Osteopatia. ${ }^{1-2}$

A musicoterapia, umas das práticas implementadas recentemente, se define como a utilização da música com fins terapêuticos e de tratamentos para a saúde ${ }^{1}$. Esta prática exerce grande influência nas pessoas por estimular afeto, socialização, movimentos com o corpo, além de desenvolver no ser humano seu lado criativo, emocional, sensorial como o tato e audição e entre outros sentimentos que relaxam e promovem conforto. ${ }^{1}$ A execução dessas práticas também proporciona uma articulação não verbal do paciente e assim consequentemente facilitará a expressão deste em situações diversas. ${ }^{1,3}$

A utilização da música como terapia na área na área da saúde é caracterizada como tecnologia leve e pode ser benéfica nos aspectos emocionais, na expressão da subjetividade e na melhora da adesão ao tratamento de questões físicas. ${ }^{4} \mathrm{~A}$ sua aplicação inclui não apenas 


\section{JOURNALOF

músicas, mas também sons, ritmos, melodias e harmonia, facilitando a interação entre indivíduos e profissionais. ${ }^{1,4}$

A implementação desta prática nas instituições de saúde por profissionais especializados e de forma multiprofissional tem enorme importância, considerando que é perceptível o auxílio aos pacientes em um espectro maior que seus anseios negativos. Sendo então uma prática que além de proporcionar relaxamento ao paciente, promove a melhora do acolhimento e aumento da humanização por parte dos profissionais de saúde. ${ }^{5}$

Logo, o objetivo desse estudo foi analisar e sintetizar informações quanto a utilização da música como estratégia de cuidado pelos profissionais que trabalham nos serviços de saúde.

\section{MATERIAIS E MÉTODOS}

Selecionou-se como método de investigação para compor o referencial teórico desta pesquisa a Revisão Integrativa (RI), com o propósito de identificar e analisar a produção científica publicada sobre a temática proposta. Para isso, utilizou-se os seguintes passos: 1) definição do tema e levantamento da pergunta norteadora: "Nos serviços de saúde, a música tem sido utilizada como estratégia de cuidado pelos profissionais?" 2) designação dos critérios para classificar quais trabalhos possuem as informações de interesse; 3) determinação dos dados pertinentes a serem coletados dos estudos, e organizá-los; 4) leitura crítica; 5) compreensão e avaliação dos resultados; 6) produzir uma síntese das informações coletadas. ${ }^{6}$

A primeira etapa foi realizada por pesquisadores de forma independente, selecionando artigos nas bases de dados: Literatura Latino-Americana e do Caribe em Ciências da Saúde (LILACS) e Scientific Eletronic Library Online (SciELO), utilizando os Descritores em Ciências da Saúde (DeCS) "Musicoterapia" OR "terapias complementares" AND "serviços de saúde" e para a Base de dados PubMed, foram utilizados os descritores "Music Therapy" [Mesh] AND "Health Services" [Mesh] AND "Complementary Therapies" [Mesh]".

Foram utilizados como critérios de inclusão os artigos completos, gratuitos, disponíveis nos idiomas inglês, português e espanhol, que foram publicados nos últimos 10 anos (período de setembro de 2007 a setembro de 2017), em revistas da área da saúde e Ciências Humanas; e que versavam sobre a temática proposta. Artigos que não se enquadraram nos critérios e/ou não abordaram o tema central foram excluídos, assim como, aqueles que se repetiram nas bases de dados. Após utilização dos critérios selecionou-se trinta e quatro artigos.

Para a extração dos dados dos artigos selecionados, foi utilizado o instrumento proposto por Ursi, ${ }^{7}$ o qual contempla identificação do artigo original, características metodológicas do estudo, avaliação do rigor metodológico, das intervenções mensuradas e dos resultados encontrados.

Os processos de seleção dos artigos utilizados estão detalhados na 


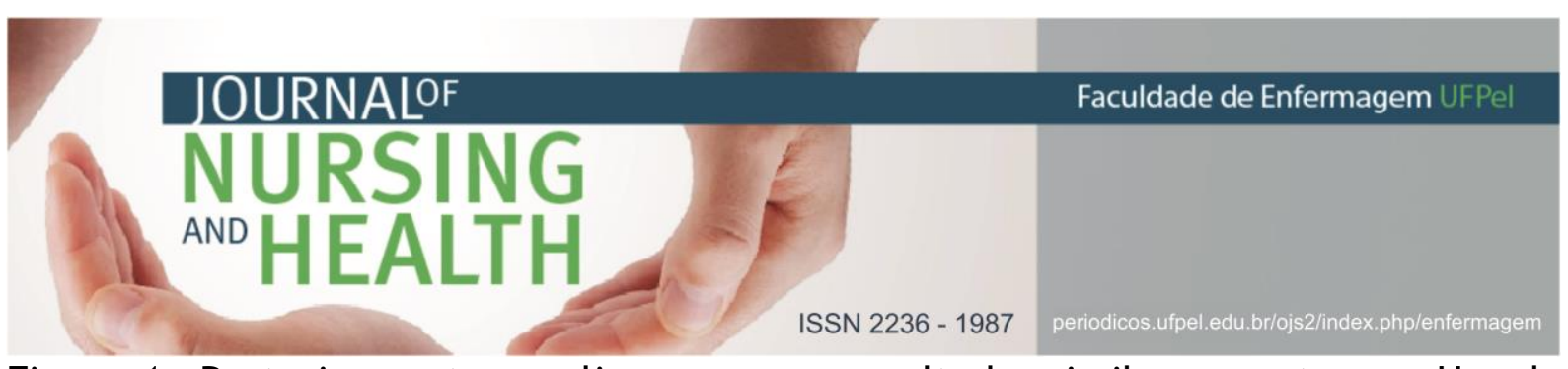

Figura 1. Posteriormente, realizou-se uma busca intensiva manual (handsearch) para uma abrangência quanto aos artigos que se enquadram no assunto proposto por esta RI. Nesse processo, ocorre a busca por artigos que entrem nos critérios de inclusão que estão citados nas referências dos textos encontrados.

Os artigos foram lidos na íntegra resultados similares em temas: Uso da Música na Ginecologia e Obstetrícia; A música e seus benefícios aos paciente e familiares; A música como ferramenta de cuidado e Prática Integrativa e Complementar utilizada na terminalidade e em pacientes psiquiátricos e o Uso da música em outros cenários da prática profissional de saúde, conforme mostra a Figura 2. e após leitura, organizaram-se os

Figura 1. Fluxograma do processo de seleção dos artigos, segundo as bases de dados e busca intensiva manual (handsearch).

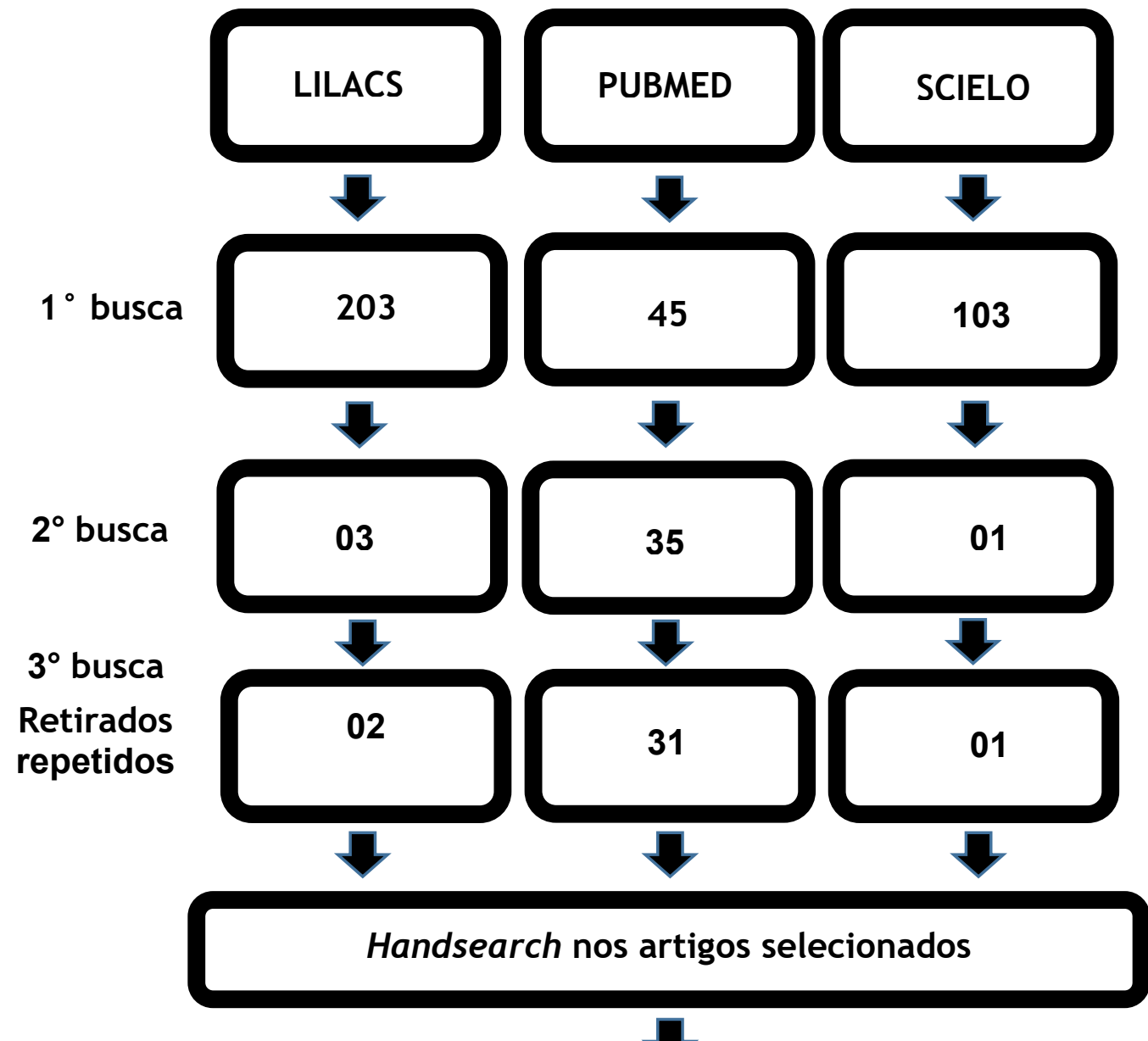

34 Artigos - Análise final e extração das informações

Fonte: dados da pesquisa, 2019. 


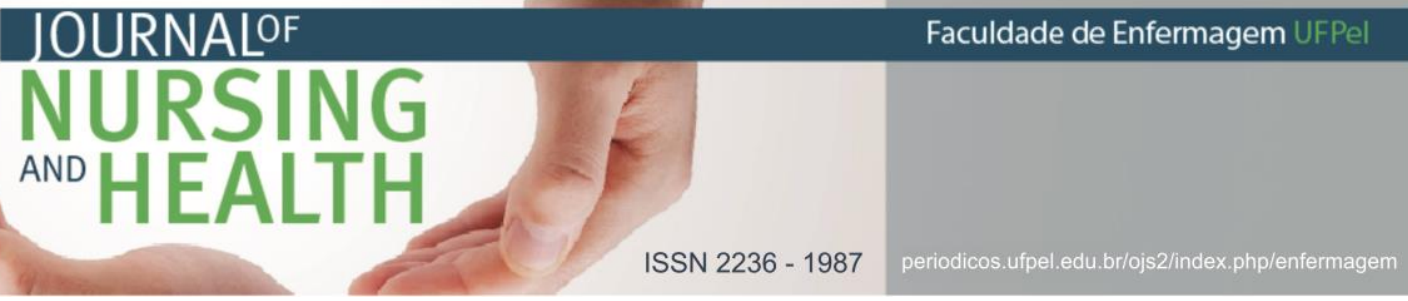

Figura 2: Categorias (núcleos de sentidos) estruturados após a leitura dos artigos.

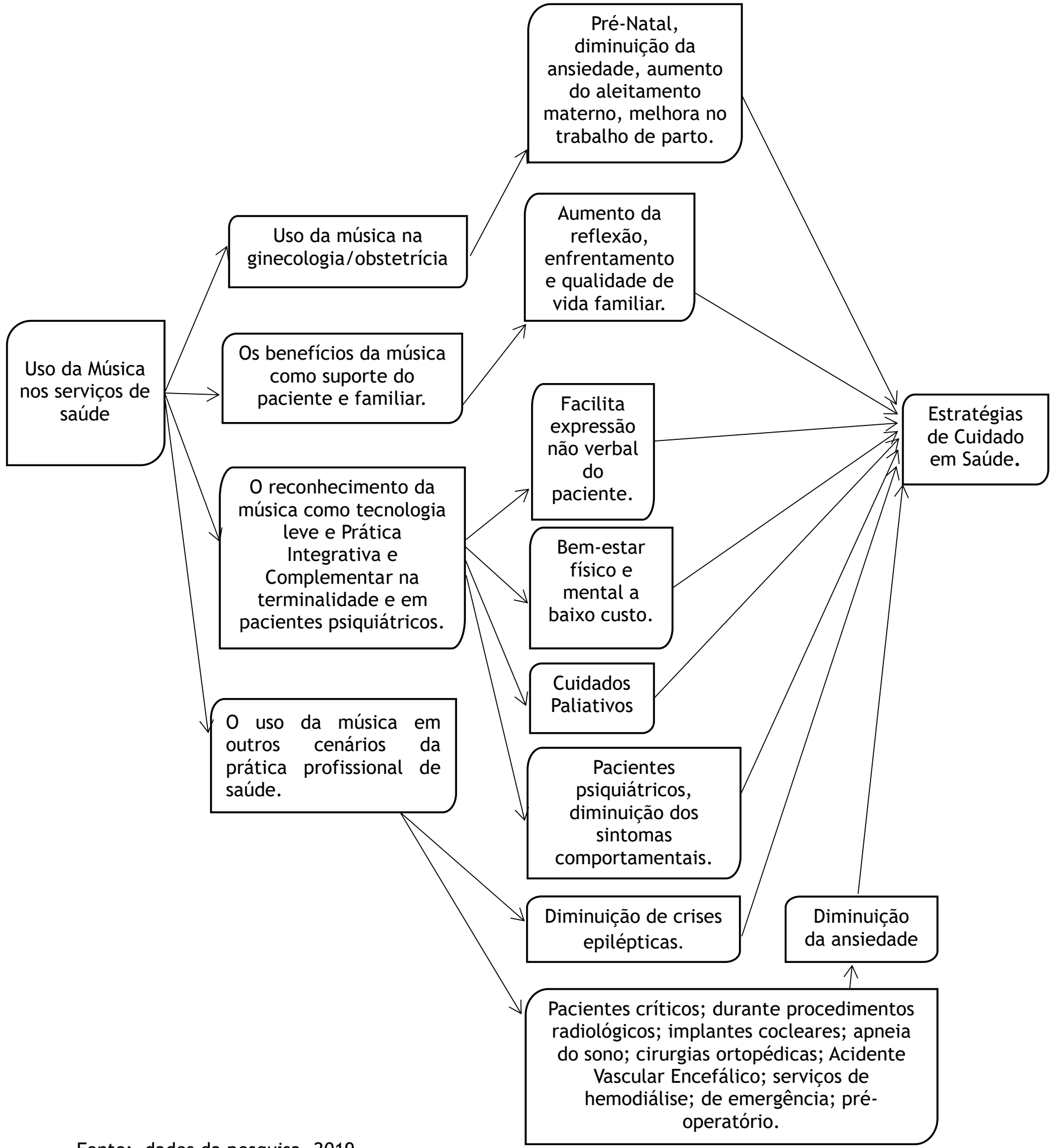

Fonte: dados da pesquisa, 2019. 


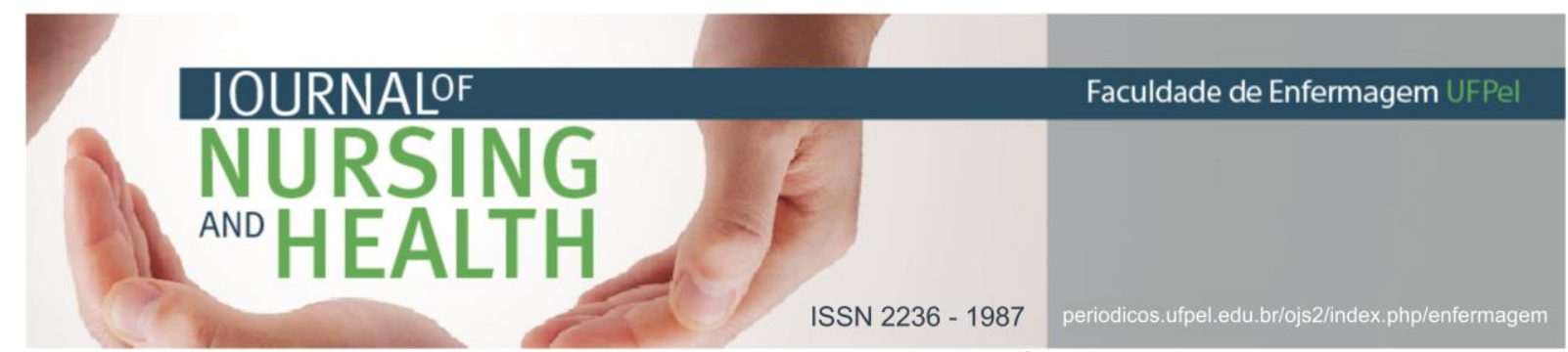

\section{RESULTADOS}

A amostragem dessa RI resultou em 34 trabalhos selecionados. Trinta e um artigos estavam na PubMed (91\%), dois artigos na SciELO (6\%) e um artigo na LILACS (3\%) totalizando 34 artigos (100\%). Em resposta à pergunta norteadora, foi observado uma baixa utilização da musicoterapia nos serviços, principalmente na América do Sul e Ásia.

Em relação ao local de publicação: 15 estudos (44\%) foram realizados na América do Norte, 10 (30\%) na Europa, sete (20\%) na América do Sul e apenas dois (6\%) na Ásia. Considerando o ano de divulgação dos artigos: nove foram publicados em 2015 (26\%), seis em 2014 (17\%), cinco em 2011 (15\%), quatro em 2012 (12\%), três em 2008 (9\%), dois em 2013 e 2016 (6\%) e um em 2007, 2009 e 2017 (3\%). 0 idioma predominante foi a língua inglesa com 26 artigos (79\%), seguido pela língua portuguesa com sete (21\%).

Ocorreu maior número de publicações que possuíam evidências adquiridas de pesquisa não experimental, de caráter descritivo ou qualitativo, delimitando-se como nível IV (53\%). Foram observadas 15 pesquisas (44\%) que apresentavam evidências adquiridas do resultado de metanálise de esboços clínicos controlados que foram randomizados e finalmente uma pesquisa (3\%) caracterizada por relato de experiência ou eventos.

Muitos artigos retratam a música como uma abordagem clínica, precisa e valorizam sua importância como estratégia de cuidado em saúde.

\section{DISCUSSÃO}

Como supracitado, foram estruturadas quatro categorias (núcleos de sentidos) que estão apresentados abaixo:

\section{Uso da Música na Ginecologia e Obstetrícia}

O uso das PICs, incluindo a música pode ser aplicado de forma benéfica em relação à promoção da segurança e qualidade de diversas áreas, como por exemplo a assistência obstétrica humanizada. ${ }^{8}$ Nas pesquisas selecionadas a música como terapia foi citada em procedimentos obstétricos e ginecológicos. ${ }^{9-10}$

Foi percebido que durante a colposcopia as pacientes que fizeram uso dessa técnica reduziram consideravelmente a ansiedade e a dor no procedimento. ${ }^{10}$ Identificou-se ainda, mesmo que discreto, os benefícios no aumento da quantidade de aleitamento materno de recémnascido pré-termo hospitalizados. ${ }^{9}$

A música foi utilizada durante o trabalho de parto com o intuito de diminuir a dor de forma não farmacológica. Logo, foi observado a necessidade de fortalecimento dos achados com novos ensaios clínicos controlados que a validem e a incorporem aos cuidados obstétricos. Identificou-se que há benefícios conversar sobre práticas integrativas no pré-natal (acupuntura, técnicas de respiração e de relaxamento muscular, crioterapia e musicoterapia) como um método não farmacológico para diminuição da dor no momento do parto, facilitando a escolha da melhor prática no momento do parto. Ao final, 


\section{JOURNALOF \\ NURSING \\ AND HEALTH}

ISSN 2236 - 1987

$\mathrm{Na}$ área médica a música proporciona aos familiares um espaço de auto expressão e facilitação da comunicação entre profissionais e pacientes envolvidos. ${ }^{4}$ Nos cuidados terminais foi evidente que a música possibilita momentos felizes, de emoção e de bem-estar, além de reforçar as práticas humanizadas nesses momentos de difícil aceitação familiar. ${ }^{22}$ A musicoterapia também proporcionou melhora nas relações familiares de pacientes que realizaram transplante de medula óssea. ${ }^{23}$

Em um estudo ${ }^{24}$ se observou que a utilização da música em crianças e adolescentes possibilita um cuidado integral dos indivíduos, fazendo com que promova bem-estar físico e mental em baixo custo. Proporciona também, um cuidado de enfermagem ampliado e que possui um baixo custo de implementação e aplicação. Frente a um processo de cuidar complexo, multifatorial e humanizado, a música se mostra um instrumento de intervenção que transcende a questão individual. Evidencia sua efetividade, também, na melhora da comunicação com a família, que se envolvem tanto quanto $o$ indivíduo no processo de promoção e recuperação da saúde.

A música como ferramenta de cuidado e Prática Integrativa e Complementar utilizada na terminalidade e em pacientes psiquiátricos

A música além de ferramenta de cuidado, pode ser considerada uma tecnologia leve de saúde, que se caracteriza como as relações de interação humana provenientes de um trabalho vivo em ato, assim 


\section{JOURNALOF \\ NURSING \\ AND HEALTH}

ISSN $2236-1987$

Algumas pesquisas demonstraram a aplicabilidade do uso dessa prática integrativa em pacientes psiquiátricos. ${ }^{31-35}$ Um dos autores identificou a musicoterapia em uso para tratamento dos sintomas da demência, observando que a evidência ainda é de baixa qualidade para se afirmar os benefícios dessa prática. ${ }^{31}$ Outro estudo identificou uma redução significativa dos sintomas comportamentais e psicológicos de pacientes com o mesmo transtorno, aflorando benefícios sobre questões de identidade pessoal e eventos que aconteceram durante a sua vida. ${ }^{33}$

Nos Estados Unidos, realizou um levantamento em 591 hospitais psiquiátricos aleatórios, nos quais encontraram que as instituições que mais se destacaram na utilização da técnica, foram as de grande porte e as que não possuíam finalidade lucrativa. Foi perceptível que menos de um terço dos hospitais psiquiátricos do país oferecem serviços alternativos, como por exemplo a musicoterapia, que proporciona aos indivíduos e familiares diversos benefícios relacionados ao enfrentamento de dor e da qualidade de vida em geral. ${ }^{35}$ Outro estudo ${ }^{36}$ relacionou a música como terapia às atividades de fisioterapia que foram realizadas em um grupo de idosos que possuíam Parkinson. Dentre os fatores relacionados, podem-se observar diferenças significativas em relação ao equilíbrio, velocidade da marcha, número de passos e tempo da distância percorrida. Em contrapartida, não foi observada variação nos estados de atividade de vida diária e motora. 


\section{JOURNALOF \\ NURSING

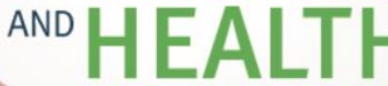

ISSN $2236-1987$

analisar a efetividade do uso da música em doenças renais crônicas, pois, essas comprovam quanti e qualitativamente a sua eficácia e contribuições para os indivíduos. ${ }^{46}$

Em outra pesquisa ${ }^{27}$ identificouse a musicoterapia dentre as mais frequentes práticas complementares utilizadas nos serviços de saúde, além de apontar seus benefícios no controle da ansiedade, depressão e dor, influenciando diretamente na qualidade de vida dos indivíduos. A saúde é uma área que se caracteriza por uma enorme complexidade de fatores determinantes, sendo assim são necessárias ações conjuntas, integradas e uma busca constante de melhorias. A utilização da música pode ser uma ferramenta viável neste processo. ${ }^{50}$

\section{CONCLUSÃO}

observou uma diminuição do número de crises epilépticas, sendo consideráveis os benefícios da música nessa patologia, quando somada ao tratamento medicamentoso. Os mecanismos desses achados ainda são obscuros, porém remontam à hipotética ativação do sistema dopaminérgico e também uma relação com os neurônios espelho. ${ }^{48}$

Foi observado a eficácia da música em pacientes pré-operatórios, nos quais a ansiedade é claramente diminuída após a aplicação da técnica. ${ }^{19} \mathrm{Em}$ uma investigação, ${ }^{49}$ por sua vez, não há nenhuma variação significativa nos pacientes cirúrgicos que tiveram contato com a música, principalmente em relação às variáveis de ansiedade e dor.

Contudo, são essenciais os estudos como ensaios clínicos para
Os estudos conduzidos principalmente em países da América do Sul e Ásia demonstram-se escassos, destacando a importância de maiores estudos na área. Considerando, também, a necessidade do aumento da utilização da música nos serviços de obstetrícia, psiquiatria e cirurgia. Há necessidade de ampliar o uso da técnica nas diversas áreas da saúde, pois mostrou-se benéfica em várias circunstâncias, como na diminuição da dor, da ansiedade, em sintomas psiquiátricos e no aumento de aleitamento materno.

Nos trabalhos encontrados, a música, como Prática Integrativa e Complementar em saúde, foi reconhecida pelos profissionais como ferramenta de humanização, tecnologia leve e aplicável nos diversos 


\section{JOURNALOF \\ NURSING \\ ANO HEALTH}

ISSN 2236 - 1987

cenários de prática profissionais com baixos custos. Ainda, a utilização desta técnica foi maior nas instituições hospitalares de diversos enfoques de cuidado, como citado anteriormente.

Em relação ao nível de evidência, pode-se observar que a maioria das metodologias se caracterizou por pesquisas quanti-qualitativas e relatos de experiência, porém, uma grande parte dos estudos foram de estudos randomizados, ensaios clínicos, o que evidencia a relação de causa e efeito da música na saúde das pessoas e aumenta o grau de evidência dos estudos.

Observou-se ainda amostras de pesquisas compostas por grupos: geriátrico, pediátrico, obstétrico e ginecológico, psiquiátrico e em grupo de adultos em geral. No entanto, ainda existem poucas pesquisas encontradas principalmente com indivíduos idosos necessitando de mais trabalhos nessa área.

\section{REFERÊNCIAS}

1 Ministério da Saúde (BR). Conselho Nacional de Saúde. Portaria $n^{\circ} 849$, de 27 de março de 2017: inclui a arte terapia, ayurveda, biodança, dança circular, meditação, musicoterapia, naturopatia, osteopatia, quiropraxia, reflexoterapia, reiki, shantala, terapia comunitária integrativa e yoga à política nacional de práticas integrativas e complementares [Internet]. Brasília; 2017[acesso em 2019 abr 04]. Disponível em: http://bvsms. saude.gov.br/bvs/saude legis/gm/2017/prt0849_28_03_2017.h tml
2 Ministério da Saúde (BR). Política Nacional de Práticas Integrativas e Complementares em Saúde [Internet]. Brasília: Ministério da Saúde; 2012 [acesso em 2018 nov 08]. Disponível em:

http: / /dab.saude.gov.br/portaldab/a pe_pic.php?conteudo=onde_tem_pics.

3 Monteiro DHM, Fermoseli AFO. Musicoterapia: contribuição como ferramenta terapêutica no auxílio a tratamentos de patologias adversas inseridas no âmbito da saúde. Cad. Grad. Ciênc. Hum. Soc. Unit (Impr.). [Internet]. 2014[acesso em 2018 nov 08];2(2):1-19. Disponível em: https://periodicos.set.edu.br/index.p $\mathrm{hp} /$ fitsbiosaude/article/view/1547/10 46.

4 Barcellos LRM. Musicoterapia em medicina: uma tecnologia leve na promoção da saúde - a dança nas poltronas! Revista música hodie [Internet]. 2015[acesso em 2018 nov 08];15(2). Disponível em: https://www.revistas.ufg.br/musica/ article/view/39679/20244.

5 Caitano JSO, Azevedo EB, Costa LFP, Soares CCD, Aguiar PV, Filha MOF. Música durante 0 transoperatório: concepção de profissionais e pacientes. Rev. bras. pesqui. saúde. [Internet]. 2014[Acesso em 2018 nov 08];16(2):76-83. Disponível em: http://periodicos.ufes.br/RBPS/articl e/view/9289/6464.

6 Mendes KDA, Silveira RCRP, Galvão CM. Revisão integrativa: método de pesquisa para a incorporação de evidências na saúde e na enfermagem. Texto \& contexto enferm. [Internet]. 2008[acesso em 2018 nov 08];17(4):758-64. Disponível em: 


\section{JOURNALOF \\ NURSING \\ AND 는 \\ ISSN 2236 - 1987}

http://www.scielo.br/pdf/tce/v17n4/ 18.pdf.

7 Ursi ES, Gavão CM. Prevenção de lesões de pele no perioperatório: revisão integrativa da literatura. Rev. latinoam. enferm. (Online). [Internet]. 2006[acesso em 2018 nov 08];14(1):124-31. Disponível em: http://www.scielo.br/pdf/rlae/v14n1 /v14n1a17.pdf.

8 Sartori AL, Vieira F, Almeida NAM, Bezerra ALQ, Martins CA. Estrategias no farmacológicas para aliviar el dolor durante el proceso del parto. Enferm. glob. [Internet]. 2011[acesso em 2018 Nov 08];10(21):1-9. Disponível em: http://scielo.isciii.es/pdf/eg/v10n21/ revision4.pdf.

9 Vianna MNS, Barbosa AP, Carvalhes AS, Cunha AJLA. Music therapy may increase breastfeeding rates among mothers of premature newborns: a randomized controlled trial. J. pediatr. (Rio J.). [Internet]. 2011 [cited 2018 Nov 08];87(3):206-12. Available from: http://www.scielo.br/pdf/jped/v87n 3/en_a05v87n03.pdf.

10 Galaal K, Bryant A, Deane KH, Alkhaduri M, Lopes AD. Interventions for reducing anxiety in women undergoing Colposcopy. Cochrane Database Syst Rev [Internet]. 2014[cited 2018 Nov 09];(12):CD006013. Available from: https://www.ncbi.nlm.nih.gov/pmc/a rticles/PMC4161490/pdf/emss-

57591.pdf.

11 Rouster-Stevens K, Nageswaran S, Arcury TA, Kemper KJ. How do parents of children with juvenile idiopathic arthritis (JIA) perceive their therapies? BMC Complement Altern Med [Internet]. 2008[cited 2018 Nov
09];8(25):1-9.

Available

from: https: / /www.ncbi.nlm.nih.gov/pmc/a rticles/PMC2424030/pdf/1472-6882-825.pdf.

12 Schlez A, Litmanovitz S, Bauer T, Dolfin R, Regev R, Arnon S. Combining kangaroo care and live harp music therapy in the neonatal intensive care unit setting. Isr Med Assoc J [Internet]. 2011[cited 2018 Nov 09];13(6):354-7. Available from: https://www.ima.org.il/FilesUpload/I MAJ/0/39/19874.pdf.

13 Silva CM, Cação JMR, Silva KCS, Marques CF, Merey LS. Physiological responses of preterm newborn infants submitted to classical music therapy. Rev. Paul. Pediatr. (Ed. Port., Online). [Internet]. 2013 [cited 2018 Nov 09];31(1):30-6. Available from: http://www.scielo.br/pdf/rpp/v31n1 /06.pdf.

14 Loewy J, Stewart K, Dassler AM, Telsey A, Homel P. The effects of music therapy on vital signs, feeding, and sleep in premature infants. Pediatrics [Internet]. 2013[cited 2018 Nov 09];131(5). Available from: http: / / pediatrics.aappublications.org /content/131/5/902

15 Stensaeth K. "Musical cocreation'? Exploring health-promoting potentials on the use of musical and interactive tangibles for families with children with disabilities. Int J Qual Stud Health Well-being [Internet]. 2013[cited 2018 Nov 09];8(20704):112. Available from: https: / /www.ncbi.nlm.nih.gov/pmc/a rticles/PMC3740601/pdf/QHW-820704.pdf.

16 Vander Heijden MJE, Araghi SO, Jeekel J, Reiss IKM, Hunink MGM, Dijk 


\section{JOURNALOF \\ NURSING

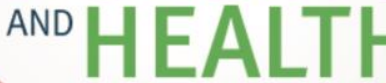

ISSN 2236 - 1987

MV. Hospitalized premature infants benefit from music interventions? a systematic review of randomized controlled trials. PLoS One [Internet]. 2016[cited 2018 Nov 09];11(9). Available from: https: / / journals.plos.org/plosone/arti cle?id=10.1371/ journal.pone.0161848.

17 Silva VA, Marco SS, Sales CA. Percepções de familiares de pessoas portadoras de câncer sobre encontros musicais durante 0 tratamento antineoplásico. Rev. bras. enferm. [Internet]. 2014[acesso em 2018 nov 08];67(3):408-14. Disponível em: http: / /www.scielo.br/pdf/reben/v67 n3/0034-7167-reben-67-03-0408.pdf.

18 Greenlee H, Balneaves LG, Carlson LE, Cohen M, Deng G, Hershman D, et al. Clinical practice guidelines on the use of integrative therapies as supportive care in patients treated for breast cancer. J Natl Cancer Inst Monogr [Internet]. 2014[cited 2018 Nov 08];2014(50):346-58. Available from: https: //www.ncbi.nlm.nih.gov/pmc/a rticles/PMC4411539/pdf/lgu041.pdf.

19 Palmer JB, Lane D, Mayo D, Schluchter $M$, Leeming R. Effects of music therapy on anesthesia requirements and anxiety in women undergoing ambulatory breast surgery for cancer diagnosis and treatment: a randomized controlled trial. Journal of clinical oncology [Internet]. 2015[cited 2018 Nov 08];33(28):3162-8. Available from:

https: / /www.ncbi.nlm.nih.gov/pmc/a rticles/PMC4979095/pdf/zlj3162.pdf.

20 Burns DS, Perkins SM, Tong Y, Hilliard RE, Cripe LD. Music therapy is associated with family perception of more spiritual support and decreased breathing problems in cancer patients receiving hospice care. J Pain Symptom Manage [Internet]. 2015[cited 2018 Nov 08];50(2):1-12. Available from: https://scholarworks.iupui.edu/bitstr eam/handle/1805/7804/Burns-2015Music.pdf?sequence=1\&isAllowed=y.

21 Mendes MVS, Cavalcante AS, Oliveira EF, Pinto DMR, Barbosa TSM, Camargo CL. Children with neuropsychomotor development delay: music therapy promoting quality of life. Rev. bras. enferm. [Internet]. 2015[cited 2018 Nov 08];68(5):515-20. Available

from: http://www.scielo.br/pdf/reben/v68 n5/0034-7167-reben-68-05-0797.pdf.

22 Sales CA, Silva VA, Pilger C, Marcon SS. Music in human terminality: the family members' conceptions. Rev. Esc. Enferm. USP. [Internet]. 2011[cited 2018 Nov 08];45(1):134-40. Available

from: http: / / www.scielo.br/pdf/reeusp/v45 n1/en_19.pdf.

23 Doro MP, Pelaez JM, Dóro CA, Antonechen AC, MalvezziM, Bonfim CMS, et al. Psicologia e musicoterapia: uma parceria no processo psicoativo dos pacientes do serviço de transplante de medula óssea. Rev. SBPH. [Internet]. 2015[acesso em 2018 nov 08];18(1):105-30. Disponível em: http://pepsic.bvsalud.org/pdf/rsbph/ v18n1/v18n1a06.pdf.

24 Silva LAGP, Baran FDP, Mercês NNA. Music in the care of children and adolescents with cancer: integrative review. Texto \& contexto enferm. [Internet]. 2016[cited 2018 Nov 08];25(4):E1720015. Available from: http://www.scielo.br/pdf/tce/v25n4/ 0104-0707-tce-25-04-1720015.pdf. 
25 Silva DC, Alvim NAT, Figueiredo PA. Tecnologias leves em saúde e sua relação como cuidado de enfermagem hospitalar. Esc. Anna Nery Rev. Enferm. [Internet]. 2008[acesso em 2019 mar 20];12(2):291-8. Disponível em:

http://www.scielo.br/pdf/ean/v12n2 /v12n2a14.

26 Ferri SMN, Pereira MJB, Mishima SM, Caccia-Bava MCG, Ameida MCP. As tecnologias leves como geradoras de satisfação em usuários de uma unidade de saúde da família. Interface comun. saúde educ. [Internet]. 2007[acesso em 2019 mar 20]; 11(23):515-29. Disponível em: http://www.scielo.br/pdf/icse/v11n2 3/a09v1123.pdf.

27 Caires JS, Andrade TA, Amaral JB, Calasans MTA, Rocha MDS. The use of complementary therapies in palliative care: benefits and purposes. Cogitare enferm. [Internet]. 2014[cited 2018 Nov 08];19(3):471-7. Available from: https://revistas.ufpr.br/cogitare/arti cle/view/33861/23227.

28 Warth M, Kessler J, Kotz S, Hillecke TK, Bardenheuer HJ. Effects of vibroacoustic stimulation in music therapy for palliative care patients: a feasibility study. BMC Complement Altern Med [Internet]. 2015[cited 2018 Nov 08];15(436):1-8. Available from: https://www.ncbi.nlm.nih.gov/pmc/a rticles/PMC4681146/pdf/12906_2015_ Article_933.pdf.

29 Warth M, KeBler J, Hillecke TK, Bardenheuer HJ. Music therapy in palliative care-a randomized controlled trial to evaluate effects on relaxation. Dtsch Arztebl Int [Internet]. 2015[cited 2018 Nov
08];112(46):788-94. Available from: https://www.ncbi.nlm.nih.gov/pmc/a rticles/PMC4671329/pdf/Dtsch_Arzteb I_Int-112-0788.pdf.

30 Preissler $\mathrm{P}$, Kordovan S, Ullrich A, Bokemeyer C, Oechsle K. Favored subjects and psychosocial needs in music therapy in terminally ill cancer patients: a content analysis. BMC Palliat Care [Internet]. 2016[cited 2018 Nov 08];15(48):1-9. Available from:

https: / /bmcpalliatcare. biomedcentral .com/articles/10.1186/s12904-0160122-7.

31 Butler R, Radhakrishnan R. Dementia. BMJ Clin Evid [Internet]. 2012[cited 2018 Nov 08];09(1001):127. Available from: https://www.ncbi.nlm.nih.gov/pmc/a rticles/PMC3437526/pdf/20121001.pdf.

32 Geretsegger M, Holck U, Gold C. Randomised controlled trial of improvisational music therapy's effectiveness for children with autism spectrum disorders (TIME-A): study protocol. BMC Pediatr [Internet]. 2012[cited 2018 Nov 08];12(2):1-9. Available from: https: / /bmcpediatr.biomedcentral.co $\mathrm{m} /$ track/pdf/10.1186/1471-2431-12-

2.

33 McDermott O, Orrell M, Ridder HM. The importance of music for people with dementia: the perspectives of people with dementia, family carers, staff and music therapists. Aging Ment Health [Internet]. 2014[cited 2018 Nov 08];18(6):706-16. Available from: https://www.ncbi.nlm.nih.gov/pmc/a rticles/PMC4066923/pdf/camh18_706. pdf. 
34 Lin LC, Lee MW, Wei RC, Mok HK, Yang RC. Mozart K.448 listening decreased seizure recurrence and epileptiform discharges in children with first unprovoked seizures: a randomized controlled study. BMC Complement Altern Med [Internet]. 2014[cited 2018 Nov 08];14(17):1-8. Available from: https: //www.ncbi.nlm.nih.gov/pmc/a rticles/PMC3893543/pdf/1472-688214-17.pdf.

35 Dain AS, Bradley EH, Hurzeler R, Aldridge MD. Massage, music and art therapy in hospice: results of a national survey. J Pain Symptom Manage [Internet]. 2015[cited 2018 Nov 08];49(6):1-14. Available from: https://www.ncbi.nlm.nih.gov/pmc/a rticles/PMC4480160/pdf/nihms670656 .pdf.

36 Yamashita FC, Saito TC, Almeida IA, Barboza NM, Santos SMS. Efetividade da fisioterapia associada à musicoterapia na doença de Parkinson. Conscientiae saúde (Impr.). [Internet]. 2012[acesso em 2018 nov 08];11(4):677-84. Disponível em: https: / / www.redalyc.org/html/929/9 2924959019/

37 Chlan LL, Savik K. Contributors to fatigue in patients receiving mechanical ventilatory support: a descriptive correlational study. Intensive Crit Care Nurs [Internet]. 2015[cited 2018 Nov 09];31(5):1-13. Available from: https://www.ncbi.nlm.nih.gov/pmc/a rticles/PMC4577301/pdf/nihms680682.pdf.

38 Chlan LL, Weinert CR, Heiderscheit A. Effects of patient-directed music intervention on anxiety and sedative exposure in critically ill patients receiving mechanical ventilatory support: a randomized clinical trial. JAMA [Internet]. 2013[cited 2019 Apr 19];309(22):2335-44. Available from: https://jamanetwork.com/journals/ja ma/fullarticle/1687827

39 DellaVolpe JD, Huang DT. Is there a role for music in the ICU? Critical care [Internet]. 2015[cited 2018 Nov 09];19(17):1-3. Available from: https: / /www.ncbi.nlm.nih.gov/pmc/a rticles/PMC4296542/pdf/13054_2014_ Article_663.pdf.

40 Kulkarni S, Johnson PCD, Kettles S, Kasthuri RS. Music during interventional radiological procedures, effect on sedation, pain and anxiety: a randomised controlled trial. Br J Radiol [Internet]. 2012[cited 2018 Nov 09];85(1016):1059-63. Available from: https: / /www.ncbi.nlm.nih.gov/pmc/a rticles/PMC3587064/pdf/bjr-851059.pdf.

41 Henry JA, Zaugg TL, Myers PJ, Schechter MA. Using therapeutic sound with progressive audiologic tinnitus management. Trends Amplif [Internet]. 2008[cited 2018 Nov 09];12(3):188-209. Available from: https: / /www.ncbi.nlm.nih.gov/pmc/a rticles/PMC4134892/pdf/10.1177_108 4713808321184.pdf.

42 Gfeller K, Guthe E, Driscoll V, Brown CJ. A preliminary report of musicbased training for adult cochlear implant users: rationales and development. Cochlear Implants Int [Internet]. 2015[cited 2018 Nov 09];16(03):22-31. Available from: https: / /www.ncbi.nlm.nih.gov/pmc/a rticles/PMC4646703/pdf/nihms701625 .pdf. 


\section{ISSN $2236-1987$}

43 Smith CE, Dauz E, Clements F, Werkowitch $M$, Whitman R. Patient education combined in a music and habit- forming intervention for adherence to continuous positive airway (CPAP) prescribed for sleep apnea. Patient Educ Couns [Internet]. 2009[cited 2018 Nov 09];74(2):184-90. Available from: https://www.ncbi.nlm.nih.gov/pmc/a rticles/PMC2653854/pdf/nihms92751. pdf.

44 Niu NN, Perez MT, Katz JN. A singing intervention for preoperative hypertension prior to total joint replacement: case report. Arthritis Care Res (Hoboken) [Internet]. 2011[cited 2018 Nov 09];63(4):630-32. Available from: https://www.ncbi.nlm.nih.gov/pmc/a rticles/PMC3075493/pdf/nihms-

253802.pdf.

45 Käll LB, Lundgren-Nilsson A, Blomstrand C, Pekna $M$, Pekna $M$, Nilsson $M$. The effects of a rhythm and music-based therapy program and therapeutic riding in late recovery phase following stroke: a study protocol for a three-armed randomized controlled trial. BMC Neurol [Internet]. 2012[cited 2018 Nov 09];12(141):1-13. Available from: https://www.ncbi.nlm.nih.gov/pmc/a rticles/PMC3554429/pdf/1471-237712-141.pdf.

46 Caminha LB, Silva MJP, Leão ER. The influence of musical rhythms on the perception of subjective states of adult patients on dialysis. Rev. Esc. Enferm. USP. [Internet]. 2009[cited 2018 Nov 09];43(4):918-24. Available from:

http://www.scielo.br/pdf/reeusp/v43 n4/en_a26v43n4.pdf.
47 Gatti MFZ, Silva MJP. Ambient music in the emergency services: the professionals' perception. Rev. latinoam. enferm. (Online). [Internet]. 2007[cited 2018 Nov 09];15(3):377-83. Available from: http://www.scielo.br/pdf/rlae/v15n3 /pt_v15n3a03.pdf.

48 Candido LE, Piazzetta CM. Musicoterapia e epilepsia de difícil controle. Revista incantare [Internet]. 2015[acesso em 2018 nov 08];06(02):172-85. Disponível em: http://periodicos.unespar.edu.br/ind ex.php/incantare/article/viewFile/12 72/pdf_58.

49 Hansen MM. A feasibility pilot study on the use of complementary therapies delivered via mobile technologies on Icelandic surgical patients' reports of anxiety, pain, and self-efficacy in healing. BMC Complement Altern Med [Internet]. 2015[cited 2018 Nov 08];15(92):1-12. Available from: https: / / www.ncbi.nlm.nih.gov/pmc/a rticles/PMC4383076/pdf/12906_2015_ Article_613.pdf.

50 Pimentel AF, Barbosa RM, Chagas $M$. A musicoterapia na sala de espera de uma unidade básica de saúde: assistência, autonomia e protagonismo. Interface (Botucatu, Online). [Internet]. 2011[acesso 2019 mar 24];15(38):741-54. Disponível em: https://www.scielosp.org/pdf/icse/2 011.v15n38/741-754/pt.

Data de submissão: 02/10/2018

Data de aceite: 25/03/2019

Data de publicação: 25/04/2019 